\title{
THE ORTHOMODULAR IDENTITY AND METRIC COMPLETENESS OF THE COORDINATIZING DIVISION RING
}

\author{
RONALD P. MORASH
}

Abstract. Let $F$ be any division subring of the real quater nions $H$. Let $l_{2}(F)$ denote the linear space of all square summable sequences from $F$ and let $L$ denote the lattice of all " $\perp$-closed" subspaces of $l_{2}(F)$, where " $\perp$ " denotes the orthogonality relation derived from the $H$-valued form $(a, b)=\sum_{i=1}^{\infty} a_{i} \bar{b}_{i}$ where $a, b \in l_{2}(F)$, $a=\left(a_{i} ; i=1,2, \cdots\right)$ and $b=\left(b_{i} ; i=1,2, \cdots\right)$. Then $L$ is complete, orthocomplemented, $M$-symmetric, irreducible, atomistic, and separable, but $L$ is orthomodular if and only if $F$ is either the reals, the complex numbers, or the quaternions.

The lattice of all closed subspaces of infinite-dimensional, separable, complex Hilbert space has these seven lattice-theoretic properties:

(i) complete $[1$, p. 6];

(ii) orthocomplemented $[1$, p. 52], [2, p. 42];

(iii) atomistic (Every element is the join of the atoms beneath it.) [3, p. 48];

(iv) irreducible (The center consists precisely of 0 and 1.) $[1, p$. 67], [3, p. 27];

(v) separable (An orthogonal family of atoms has at most countably many elements.) and infinite dimensional [3, p. 58];

(vi) $M$-symmetric (If $a, b \in L$, we write $a M b$ if $x \leqq b$ implies $x \vee(a \wedge b)=(x \bigvee a) \wedge b . L$ is $M$-symmetric if $a M b$ implies $b M a$.) [1, p. 82], [3, p. 2];

(vii) orthomodular (If $a, b \in L$ and $a \leqq b$, then $b=a \vee\left(b \wedge a^{\prime}\right)$.) $[1$, p. 53$],[2$, p. 42$]$.

Real and quaternionic Hilbert space have the same properties. The question arises whether these are the only three lattices (up to ortho-isomorphism) having them. The problem underlying this question is one of coordinatization, that is, the realization of an abstract lattice, described only by algebraic properties, as a lattice associated in some natural way with a concrete object, for example, the lattice of projections of Hilbert space. Work towards a coordinatization theorem for lattices with the above properties has been done

Received by the editors May 4, 1970.

AMS 1969 subject classifications. Primary 0640; Secondary 8106.

Key words and phrases. Orthomodular lattices, coordinatization. 
by MacLaren [4], [5] and Zierler [6]. The former showed that if $L$ has properties (i) through (vi) and if $\operatorname{dim} L \geqq 4$, then $L$ is ortho-isomorphic to the lattice of closed subspaces of a semi-inner-product space over some division ring $D$. Our question is whether the assumption (vii) is enough to force $D$ to be either the reals, the complex numbers, or the quaternions. An answer of "yes" would characterize completely, in terms of lattice-theoretic properties, projection lattices of Hilbert space and would thus be of great importance in the study of the logical foundations of quantum mechanics [7, p. 71]. We present here some evidence in support of the possibility of an affirmative answer. Let $F$ be any division subring of the real quaternions $H$. We denote by $|x|=\left(a^{2}+b^{2}+c^{2}+d^{2}\right)^{1 / 2}$ the norm and by $\bar{x}=a-b i-c j-d k$ the conjugate of $x=a+b i+c j+d k \in H$. The map $x \rightarrow \bar{x}$ is an involutory anti-automorphism of $H$. Consider $l_{2}(F)$, the linear space of square-summable (with respect to the above norm) sequences from $F$. Define a definite, Hermitian, conjugate-bilinear "form" on $l_{2}(F),():, l_{2}(F) \times l_{2}(F) \rightarrow H$, by the rule $(x, y)=\sum_{n=1}^{\infty} x_{n} \bar{y}_{n}$, where $x, y \in l_{2}(F), x=\left(x_{n} ; n=1,2, \cdots\right)$ and $y=\left(y_{n} ; n=1, \cdots\right)$. Note that this form is $H$-valued, but not necessarily $F$-valued. For each subset $M$ of $l_{2}(F)$, define $M^{\perp}=\left(y \in l_{2}(F):(x, y)=0\right.$ for each $x \in M)$. Call a subspace $S$ of $l_{2}(F)$ closed in case $S=S^{\perp \perp}$. The map $S \rightarrow S^{\perp \perp}$ of the lattice $\bar{L}$ of all subspaces of $l_{2}(F)$ in to itself is a closure operator $[8$, p. 1518] and so the lattice $L$ of all closed subspaces is complete and orthocomplemented. It is also easily seen that this lattice is irreducible, atomistic, and separable. However:

THEOREM. $L$ is orthomodular if and only if $F=R, C$, or $H$.

Proof. Only the "only if" part of the theorem needs proof. We give the proof for the case $F \subseteq R$ only (that is, $F \subseteq R$ and $L$ orthomodular imply $F=R$ ). The proofs of the other two cases (that is, $F \subseteq C$, but $F \Phi R$ and $F \subseteq H$, but $F \Phi C$ ) follow from the fact that sequential convergence in $C$ or $H$ can be characterized in terms of coordinate-wise convergence in $R$. Choose $\gamma \in R$. We shall show that, if $L$ is orthomodular, then necessarily $\gamma \in F$. Let $x_{0}=1$ and let $x_{n}$ $=n / 2^{n}, \quad n=1,2,3, \cdots$ Let $x=\left(x_{n} ; n=0,1, \cdots\right)$. Let $z_{0}$ be the greatest integer less than or equal to $\gamma \sum_{n=0}^{\infty} x_{n}^{2}$. Let $z=\left(z_{n} ; n=0,1, \cdots\right)$ where.$z_{1} z_{2} \cdots$ is the binary expansion of $\gamma \sum_{n=0}^{\infty} x_{n}^{2}-z_{0}$. Thus $\sum_{n=1}^{\infty} z_{n} / 2^{n}=\gamma \sum_{n=0}^{\infty} x_{n}^{2}-z_{0}$. Let $y_{0}=z_{0}$ and let $y_{n}=z_{n} / n$ for $n=1,2, \cdots$. Let $y=\left(y_{n} ; n=0,1, \cdots\right)$. Letting $a$ $=\operatorname{sp}(x)$ and $b=\operatorname{sp}(y), a$ and $b$ are distinct atoms in $L$ so that, by orthomodularity, $c=(a \bigvee b) \wedge a^{\perp} \neq 0$ [3, p. 291]. Necessarily, $c$ is an atom so that $c=\operatorname{sp}(\tau x+y)$ for some $\tau \in F$. But $c \leqq a^{\perp}$ so that $(\tau x+y)$ 
$\perp x$, that is,

$$
0=\sum_{n=0}^{\infty}\left(\tau x_{n}+y_{n}\right) x_{n}=\tau \sum_{n=0}^{\infty} x_{n}^{2}+\sum_{n=0}^{\infty} x_{n} y_{n} .
$$

Hence,

$$
\begin{aligned}
\tau & =-\sum_{n=0}^{\infty} x_{n} y_{n} / \sum_{n=0}^{\infty} x_{n}^{2}=\left(-z_{0}-\sum_{n=1}^{\infty} z_{n} / 2^{n}\right)\left(\sum_{n=0}^{\infty} x_{n}^{2}\right)^{-1} \\
& =-\gamma\left(\sum_{n=0}^{\infty} x_{n}^{2}\right) / \sum_{n=0}^{\infty} x_{n}^{2}=-\gamma .
\end{aligned}
$$

Since $\tau \in F$, we may conclude $\gamma \in F$, as desired.

ADDED IN PROOF. The fact that $(a \bigvee b) \wedge a^{\perp} \neq 0$ for distinct atoms $a, b$ also follows from $M$-symmetry, so the theorem remains valid if we replace "orthomodular" by " $M$-symmetric." Hence, this $L$ is orthomodular if and only if it is $M$-symmetric. It follows also that the closure operation $M \rightarrow M^{\perp \perp}$ is Mackey [8, p. 1518] only when $F=R, C$, or $H$.

\section{REFERENCES}

1. G. Birkhoff, Lattice theory, 3rd ed., Amer. Math. Soc. Colloq. Publ., vol. 25, Amer. Math. Soc., Providence, R. I., 1967. MR 37 \#2638.

2. S. S. Holland, Jr., The current interest in orthomodular lattices, Trends in Lattice Theory, Van Nostrand, Princeton, N. J., 1969.

3. S. Maeda, Theory of symmetric lattices, University of Massachusetts, Amherst, Mass., 1968 (lecture notes-unpublished).

4. M. D. MacLaren, Atomic orthocomplemented lattices, Pacific J. Math. 14 (1964), 597-612. MR $29 \# 1159$.

5. - Notes on axioms for quantum mechanics, Argonne National Lab. Report ANL 7065, July 1965.

6. N. Zierler, Axioms for non-relativistic quantum mechanics, Pacific J. Math. 11 (1961), 1151-1169. MR 25 \#4385.

7. G. W. Mackey, Mathematical foundations of quantum mechanics: A lecture-note volume, Benjamin, New York, 1963. MR 27 \#5501.

8. S. S. Holland, Jr., Partial solution to Mackey's problem about modular pairs and completeness, Canad. J. Math. 21 (1969), 1518-1525.

University of Massachusetts, AmHerst, Massachusetts 01002 